\title{
Systematic theoretical uncertainties in jet quenching due to gluon kinematics
}

\author{
W. A. Horowitz ${ }^{1, *}$ and B. A. Cole $2, \dagger$ \\ ${ }^{1}$ Physics Department, The Ohio State University, 191 West Woodruff Avenue, Columbus, Ohio 43210, USA \\ ${ }^{2}$ Physics Department, Columbia University, 538 West 120th Street, New York, New York 10027, USA
}

(Received 9 October 2009; published 17 February 2010)

\begin{abstract}
We find that the current radiative energy loss kernels obtained from the opacity expansion dramatically violate the collinear approximation used in their derivation. By keeping only the lowest order in collinearity terms, models based on the opacity expansion have $\sim 50 \%$ systematic uncertainty in the calculation of $\pi^{0} R_{A A}$ in the most central RHIC collisions, resulting in a systematic uncertainty of $\sim 200 \%$ in the extracted medium density. Surprisingly, the inclusion of a thermal gluon mass of the order of the Debye screening scale affects $R_{A A}$ only at about the 5\% level due to nonintuitive coherence effects. For some observables such as $R_{A A}$, the effect of these uncertainties decreases with increasing jet energy; for others, such as the average number of radiated gluons, the effect is energy independent. We note that it is likely that the differences reported in the extracted values of medium parameters such as $\hat{q}$ by various jet energy loss models will fall within this collinear approximation systematic uncertainty; it is imperative for the quantitative extraction of medium parameters or the possible falsification of the hypothesis of weak coupling between the hard probes and the soft modes of the quark-gluon plasma medium that future radiative energy loss research push beyond the lowest-order collinear approximation.
\end{abstract}

DOI: 10.1103/PhysRevC.81.024909

PACS number(s): 12.38.Mh, 24.85.+p, 25.75.-q

\section{INTRODUCTION}

Jet quenching is a unique observable in ultrarelativistic heavy-ion collisions (URHIC) as high-transverse-momentum (high- $p_{T}$ ) particles are the most controlled, calibrated, and direct probe of the fundamental soft degrees of freedom of quark-gluon plasma (QGP) [1]. Rigorous falsification or confirmation of fundamentally different qualitative pictures of the basic physics of QGP (e.g., whether it is strongly or weakly coupled [2], its relevant degrees of freedom [3-5], etc.) from comparing theoretical predictions to high- $p_{T}$ data crucially requires a detailed understanding of both experimental and theoretical uncertainties.

Once the qualitative picture is fixed, jet tomography [6,7], the quantitative determination of bulk properties of the QGP through the study of the attenuation pattern of high-momentum particles, becomes possible. Jet tomography requires both high-precision experimental data and a precise theoretical understanding of partonic energy loss. Recent statistical analyses by PHENIX [8,9], assuming infinite precision for theoretical calculations that assume a weakly coupled QGP, suggest that data are now certain enough to extract medium properties to within $\sim 20 \%$. Given that no current perturbative energy loss model satisfactorily and simultaneously describes more than one high- $p_{T}$ observable [10,11], such as the level of suppression and azimuthal anisotropy of the high- $p_{T}$ gluons, light quarks, and heavy quarks as measured through the light meson [12-15] and nonphotonic electron nuclear modification factor [16-19], $R_{A A}\left(p_{T}, \phi\right)$, and the away-side suppression of jet-triggered hadrons, $I_{A A}$ [20], it is, perhaps, premature to claim that even a qualitative understanding of the jet quenching

\footnotetext{
*horowitz@mps.ohio-state.edu
}

†cole@nevis.columbia.edu of QGP exists. Nevertheless, continuing with the assumption that current perturbative quantum chromodynamics (pQCD) models accurately describe the physics, such quantitative extractions of medium properties can only be meaningful when the systematic uncertainties in the theoretical calculations and modeling are both known and included in the systematic uncertainties in the extracted quantities. While there have been some previous qualitative estimates of the theoretical uncertainty stemming from the running of the strong coupling [21,22], the probability leakage of the Poisson convolution [23,24], and a phenomenological infrared (IR) cutoff imposed to approximate the effects of a nonzero, thermal

TABLE I. Comparison of the extracted $d N_{g} / d y$ for the work considered here compared to PHENIX 0\%-5\% most central $\pi^{0}$ data. Note especially the difference of nearly a factor of 3 in the last three columns when using radiative energy loss (rad) only. Due to the use of the collinear approximation, all three of these values should be considered equally valid determinations of the medium density. The assumed infinite precision for the elastic channel makes the convolved radiative + elastic $(\mathrm{rad}+\mathrm{el})$ extraction of $d N_{g} / d y$ suffer a smaller systematic uncertainty than those studied in the radiative channel alone. Columns 2 and 3 demonstrate the limited influence of the radiated gluon mass in measuring the medium density. See text for more details.

\begin{tabular}{|c|c|c|c|c|c|}
\hline \multirow{3}{*}{$\begin{array}{l}x: \\
\theta_{\max }: \\
m_{g}:\end{array}$} & \multicolumn{2}{|c|}{$x_{+}$} & $x_{+}\left(x_{E}\right)$ & \multicolumn{2}{|c|}{$x_{E}$} \\
\hline & \multicolumn{4}{|c|}{$\pi / 2$} & $\pi / 4$ \\
\hline & $\mu / \sqrt{ } 2$ & \multicolumn{4}{|c|}{0} \\
\hline $\begin{array}{l}\text { Rad Only } \\
d N_{g} / d y(\times 1000)\end{array}$ & $3.0_{-0.5}^{+0.7}$ & $2.4_{-0.4}^{+0.5}$ & $2.0_{-0.3}^{+0.4}$ & $4.0_{-0.6}^{+0.9}$ & $5.9_{-1.0}^{+1.1}$ \\
\hline $\begin{array}{c}\mathrm{Rad}+\mathrm{El} \\
d N_{g} / d y(\times 1000)\end{array}$ & $1.0_{-0.1}^{+0.3}$ & $1.0_{-0.2}^{+0.2}$ & $0.9_{-0.2}^{+0.2}$ & $1.3_{-0.2}^{+0.2}$ & $1.5_{-0.3}^{+0.3}$ \\
\hline
\end{tabular}


gluon mass [25], one of the main purposes of this paper is to begin the process of rigorously quantifying the combined experimental uncertainties and systematic theoretical uncertainties in extracted medium parameters. This paper, then, is complementary to the work of Ref. [26], which demonstrated through rigorous statistical analysis the crucial importance of the choice made for the early time energy loss and the lack of sensitivity to the particular nuclear PDFs chosen.

In particular, we investigate the theoretical uncertainties due to the collinear approximation and due to different assumptions regarding the thermal mass of the radiated gluon in the opacity expansion approach [27-31] for calculating the radiative energy loss of high- $p_{T}$ partons in pQCD. We also assess how those uncertainties change when elastic energy loss is included $[32,33]$. A summary of our results is reported in Table I. Most strikingly, we find that keeping only the lowestorder term in collinearity results in an $\sim 200 \%$ systematic uncertainty in the value of the extracted gluon rapidity density for central $\mathrm{Au}+\mathrm{Au}$ collisions at top RHIC energies. While this paper does not quantify the uncertainties associated with the collinear approximation for other $\mathrm{pQCD}$ radiative energy loss models [23,27,34,35], the uncertainties in those models are almost certainly similar to the results quoted here. In particular, the sizable discrepancies between the extracted medium properties obtained by different energy loss groups $[8,9,36]$ are probably within the current theoretical systematic uncertainty.

There are, generally speaking, four main pQCD-based radiative energy loss formalisms applied to URHIC: opacity expansion (GLV) [7,27,28,30,31], multiple soft scattering (BDMPS-Z-ASW) [29,37-46], higher-twist (HT) [47-50], and thermal field theory (AMY) [51-54]. We emphasize that we are interested in quantifying the systematic theoretical uncertainty due to the approximations made in the process of deriving an energy loss formula within any current pQCDbased formalism; we do not focus on the differences due to the various physics assumptions that go into the different formalisms (this is the purview of, e.g., Ref. [36]). In this work we focus quantitatively on the opacity expansion approach; see Fig. 1 for a cartoon of the physics involved and visualizations of the key variables discussed in the text. Within

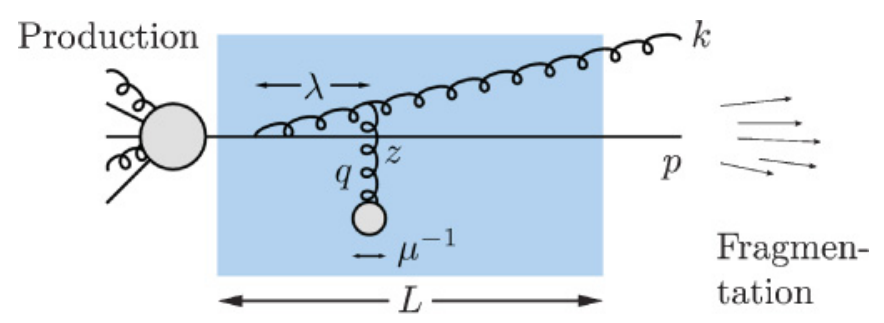

In-medium Energy Loss

FIG. 1. (Color online) Cartoon of the production, in-medium energy loss, and fragmentation processes that may occur perturbatively for a high- $p_{T}$ parton produced in a heavy-ion collision. Momentum labels are $p$ for the outgoing parent parton, $k$ for the medium-induced bremsstrahlung gluon, and $q$ for the momentum transfer between an in-medium soft degree of freedom and the high- $p_{T}$ parent parton. Note the ordering of length scales displayed, $\mu^{-1} \ll \lambda \ll L$. the GLV formalism one derives an expression for the single inclusive radiated gluon spectrum, $d N_{g} / d x$, that is folded into a Poisson convolution [7] for the distribution of total radiated energy by the parent parton (see below). Like all radiative energy loss models, the formalism makes the eikonal approximation, namely, that the parent parton has a sufficiently high energy that its path is approximately straight and that the interference from the away-side jet, $\mathcal{O}(1 / E)$, may be safely ignored [55]. The formalism also neglects contributions from four-gluon vertices and assumes that the parent parton suffers independent, path-ordered collisions. These choices are justified in the opacity expansion approach by the assumption of a medium composed of Debye-screened scattering centers whose screening length $\mu^{-1} \ll \lambda$ is much smaller than the mean free path of the parent parton [37]. Such an ordering of scales is consistent with thermal field theoretic estimates for $\mu$ and $\lambda[33,56]$.

Current evaluations of the diagrams resulting from the opacity expansion formalism drop a significant number of terms that are assumed to be small. These approximations were made for analytic simplicity, but they do not seem to be inherently required. Specifically, GLV takes: (1) the collinear approximation, $k_{T} \ll x E$, where $k$ is the momentum of the radiated gluon and $k_{T}$ its component transverse to the motion of the parent parton; (2) the parent parton path length much longer than the gluon mean free path, $L \gg \lambda$; and (3) the soft radiation limit, $x \ll 1$, where $x$ is the momentum fraction taken away by the radiated gluon (discussed further below). We note that all these assumptions are also made in the BDMPS and AMY formalisms. ${ }^{1}$ HT makes an assumption similar to (2), does not make the soft gluon approximation (3), but does assume collinearity (1). After discussing (1) in greater detail we briefly touch on (2) and (3) further.

This work focuses on the collinear approximation $k_{T} \ll x E$ and, more generally, the effects of limiting the phase space into which gluon bremsstrahlung is allowed to radiate. The current derivations using the opacity expansion formalism yield a single inclusive gluon radiation kernel, $d N_{g} / d x d k_{T}$, that knows nothing about the approximations used in its derivation; in calculating $d N_{g} / d x$ the collinear approximation is enforced phenomenologically with an ultraviolet (UV) cutoff in the $k_{T}$ integration. We find that the $d N_{g} / d x d k_{T}$ kernel maximally violates the assumption of collinearity at small $x$. It is not surprising, therefore, that $d N_{g} / d x$, and observables dependent on it such as $R_{A A}$, are highly sensitive to the $\mathcal{O}(1)$ variations of the cutoff one explores in estimating a systematic theoretical uncertainty. Even worse, as we discuss further below, the use of only the lowest-order collinear

\footnotetext{
${ }^{1}$ In GLV and BDMPS, assumption (2) is used to neglect poles from propagators multiplied by $\exp (-\mu \Delta z) \approx \exp (-\mu \lambda) \ll 1$, where $\Delta z$ is the distance between successive scattering centers; this approach is probably invalid for $L \lesssim \lambda \sim 1 \mathrm{fm}$. On the other hand, AMY uses the central limit theorem in its Langevin approach and corrections are likely for $L \lesssim 30 \lambda \sim 30 \mathrm{fm}$; this extra long path length is also required by the neglect of the interference between vacuum and inmedium induced radiation.
} 
term means that collinearly equivalent definitions of $x$ yield values of medium density extracted from data that differ by $\sim 100 \%$.

Previous work [25] that investigated a phenomenological $k_{T}$ cutoff in the IR to approximate a thermal mass of the radiated gluon found a similarly strong sensitivity to the specifics of the cutoff. However, we find that, with an explicit derivation of energy loss for nonzero gluon mass instead of a cutoff imposed by hand a posteriori, surprising and nontrivial cancellations yield an energy loss that has little sensitivity to the exact value of $m_{g}$.

\section{OTHER SOURCES OF UNCERTAINTY}

Before returning to the primary focus of the paperuncertainties introduced by the collinear approximation-it is worth enumerating here other potential sources of theoretical uncertainty that we do not attempt to quantify in this work but that, nonetheless, could be quite large.

In this paper, we calculate radiative energy loss using the first-order expression from the opacity expansion; that is, the single inclusive gluon radiation spectrum is derived by scaling up diagrams with only a single in-medium scattering for the parent parton or its bremsstrahlung radiation, as depicted in Fig. 1, by the average number of scatterings, $L / \lambda$. The $n$th order in opacity explicitly evaluates the interference terms, neglected in the lower-order expressions, from diagrams with $n$ in-medium scatterings. Within the set of approximations given previously, and assuming that the medium consists of Debye-screened static scattering centers, the GLV formalism yields a closed-form expression for $d N_{g} / d x$ to all orders in opacity. Numerical study of $d N_{g} / d x[7,28,56]$ suggests that it does receive corrections from higher orders but that these are relatively small-around the $\sim 30 \%$ level for relatively long paths, of $L=5 \mathrm{fm}$, and smaller for shorter paths-for RHICand LHC-like conditions.

The Poisson convolution [7,37] of the $d N_{g} / d x$ kernel is an attempt to approximate the full probability distribution of radiative energy loss $P(\epsilon)$, where the fraction of radiated energy is defined by $E_{f}=(1-\epsilon) E_{i}$. The convolution assumes independent, incoherent emission of gluons; the effect of neglecting the interference between two or more emitted gluons is not currently known. There are also uncertainties associated with "probability leakage" [7,23]: in model implementations the parent parton energy is often not dynamically updated throughout the convolution, leading to the possible violation of energy conservation (i.e., $P(\epsilon)$ has support for $\epsilon>1$ ). In this work, we assign the total weight from the convolution at $\epsilon>1$ to a $\delta$ function centered at $\epsilon=1$ (complete stopping), the so-called "non-reweighted" approach [23,24]. Studies have shown that this procedure reproduces reasonably well distributions obtained from Poisson convolution procedures that dynamically update the parent parton energy [57]. Moreover, for energy loss calculations based on the GLV formalism, the leakage tends to be small. When elastic energy loss is included this leakage is even smaller [58].

A realistic model of jet quenching in URHIC requires a weighted averaging over a large range of medium path lengths, $0 \lesssim L \lesssim 12 \mathrm{fm}$. QGP temperatures at RHIC energies lead to a gluonic mean free path of $\lambda \sim 1-2 \mathrm{fm}^{2}$ Additionally, even in the most central collisions, a significant portion of path lengths has $L \lesssim 2 \mathrm{fm}$, and the bias toward the surface from energy loss makes these shorter paths even more important $[23,33]$. The assumption, $L \gg \lambda$, is therefore violated for a large fraction of energy loss inducing processes. In the $R_{A A}$ calculations in this work, we simply apply the GLV formalism to all path lengths. There is an additional uncertainty associated with mapping the realistic medium density of heavy-ion collisions-with its approximate Bjorken expansion and nontrivial, time-dependent transverse density profile-into the static, uniform "brick" problem in which the analytic formulas were derived.

The small $x$ approximation seems reasonable at RHIC energies where the single inclusive gluon radiation spectrum $d N_{g} / d x$ peaks at $x \sim \mu / E \lesssim 0.05 \ll 1$. The small- $x$ approximation should apply even better at LHC, where $E$ will be larger by a factor of $\sim 10$. However, the Poisson convolution widens the original $d N_{g} / d x$ spectrum-no matter how low the $x$ value of the peak of the spectrum-thus distributing its weight out to larger values of $x$. In this way the convolution introduces a sensitivity to the large- $x$ region of $d N_{g} / d x$ that is not well controlled due to the $x \ll 1$ assumption.

Probably the largest source of uncertainty, likely even bigger than that due to the collinear approximation that we detail in this paper, will result from values chosen for and/or the running of $\alpha_{s}$ [21,22]; because radiative energy loss varies as $\alpha_{s}^{3}$, it is highly sensitive to changes in the value of the coupling. One factor of $\alpha_{s}$ arises from the emission of the gluon bremsstrahlung, while the other two result from interacting with the soft degrees of freedom in the medium; see Fig. 1. For these last two couplings it is not even clear what scale should be chosen for the running. Nevertheless, the momentum transfer $q$ will always probe soft scales of the order of $\mu \sim 0.5 \mathrm{GeV} \gtrsim \Lambda_{\mathrm{QCD}} \sim 0.2 \mathrm{GeV}$, and nonperturbative physics will be involved. The lack of a proof of factorization, whereby these nonperturbative contributions necessarily become small at high energies, is of course not particularly comforting.

\section{OPACITY EXPANSION ENERGY LOSS KERNEL AND $x$}

The first order in opacity result for the radiated gluon spectrum assuming massive quarks and gluons and using the Gyulassy-Wang model of static scattering centers [3], to lowest order in $x$ and $k_{T} / x E$, where $k_{T}=|\mathbf{k}|$, is given by [30]

$$
\begin{aligned}
x \frac{d N_{g}}{d x}= & \frac{C_{R} \alpha_{s}}{\pi} \frac{L}{\lambda} \int \frac{d^{2} \mathbf{q}}{\pi} \frac{2 d^{2} \mathbf{k}}{\pi} \frac{\mu^{2}}{\left(\mathbf{q}^{2}+\mu^{2}\right)^{2}} \\
& \times \frac{\mathbf{k} \cdot \mathbf{q}(\mathbf{k}-\mathbf{q})^{2}-\beta^{2} \mathbf{q} \cdot(\mathbf{k}-\mathbf{q})}{\left[(\mathbf{k}-\mathbf{q})^{2}+\beta^{2}\right]^{2}\left(\mathbf{k}^{2}+\beta^{2}\right)} \\
& \times \int d z\left[1-\cos \left(\frac{(\mathbf{k}-\mathbf{q})^{2}+\beta^{2}}{2 E x} z\right)\right] \rho(z) .
\end{aligned}
$$

${ }^{2}$ We follow Refs. [33] and [56] by taking $\lambda_{g}=1 / \rho_{g} \sigma_{g g}$, where $\rho_{g}=16 \zeta(3) T^{3} / \pi^{2}$ is the density of a pure glue medium and $\sigma_{g g}=$ $9 \pi \alpha_{s}^{2} / \mu^{2}$ is the tree-level $g g \rightarrow g g$ elastic scattering cross section. Note that only the gluonic mean free path is required in the opacity expansion due to color triviality [28]. 


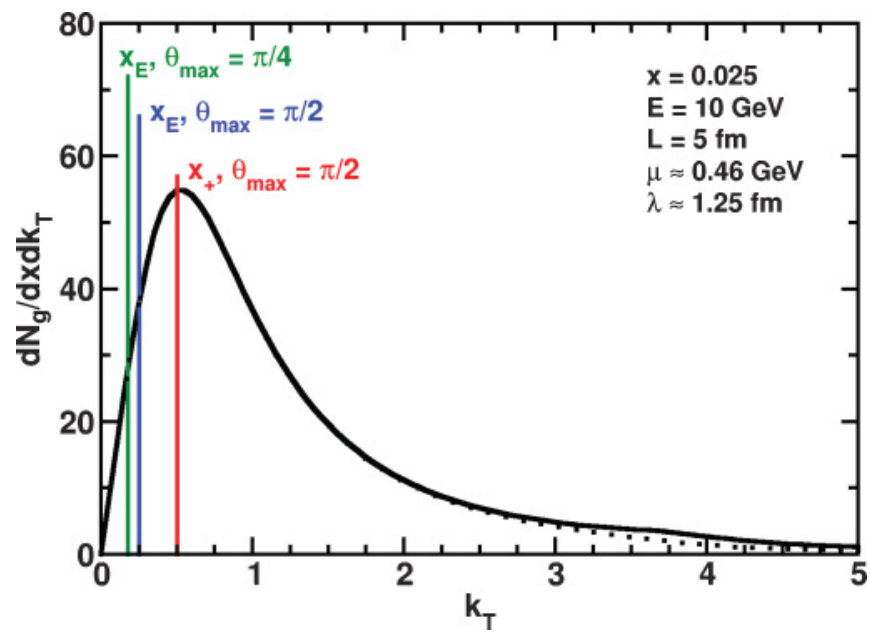

FIG. 2. (Color online) Plot of $d N_{g} / d x d k_{T}$ from Eq. (2) (solid curve) for a light quark with all masses set to $0, E=10 \mathrm{GeV}, L=$ $5 \mathrm{fm}$, and representative values of $\mu \simeq 0.46 \mathrm{GeV}$ and $\lambda \simeq 1.25 \mathrm{fm}$ for a medium density of $d N_{g} / d y=1000$, similar to RHIC conditions [33]. Vertical lines show the values of $k_{T}$ used as cutoffs to enforce collinearity in Eq. (2). Note that with $x=0.025 \sim \mu / E, d N_{g} / d x d k_{T}$ is large near $k_{T} \sim k_{\max }$, completely in contradiction with the collinear approximation. The dotted curve, from the unshifted integrand of Eq. (1), differs only slightly from the spectrum obtained from the shifted integrand of Eq. (2) (solid curve).

Here, $\beta^{2}=x^{2} M^{2}+m_{g}^{2}$, where $M$ is the mass of the parent parton and $m_{g}$ is the mass of the radiated gluon, $C_{R}=C_{F}$ for a parent quark, and $C_{R}=C_{A}$ for a parent gluon. The corresponding result for massless parent quarks/gluons and/or massless radiated gluons is obtained by setting $M$ and/or $m_{g}$ to zero in Eq. (1), respectively. After temporarily taking the $q_{T}=$ $|\mathbf{q}|$ medium exchange momentum limit to infinity and making a change of variables (see Ref. [30] for more details) and, additionally, assuming an exponentially decaying distribution, $\rho(z)$, for the distance to the scattering center, ${ }^{3}$ Eq. (1) becomes

$$
\begin{aligned}
\frac{d N_{g}}{d x}= & \frac{8 C_{R} \alpha_{s} \mu^{2}}{\pi x} \frac{L}{\lambda} \int d q_{T} d k_{T} \frac{q_{T}^{3}}{(4 x E / L)^{2}+\left(q_{T}^{2}+\beta^{2}\right)^{2}} \\
& \times \frac{k_{T}}{k_{T}^{2}+\beta^{2}} \frac{k_{T}^{2}\left(k_{T}^{2}+\mu^{2}-q_{T}^{2}\right)+\beta^{2}\left(q_{T}^{2}+\mu^{2}-k_{T}^{2}\right)}{\left[\left(k_{T}-q_{T}\right)^{2}+\mu^{2}\right]^{3 / 2}\left[\left(k_{T}+q_{T}\right)^{2}+\mu^{2}\right]^{3 / 2}} .
\end{aligned}
$$

We note that supposing the scattering center distribution to be uniform instead of exponentially decaying appears to make only a small difference in the gluon spectrum [58].

The integrand in Eq. (2) is both IR and UV safe. In principle, we could set the lower and upper $q_{T}$ and $k_{T}$ limits of integration to 0 and $\infty$, respectively. If the integrand were exact, then

\footnotetext{
${ }^{3}$ In general it is necessary to specify the distribution in the difference in distance between successive scattering centers. In the first order in opacity case, with only one scattering center, this difference in distance is between the scattering center and the production point. One can always take the production point to be at $z=0$, and thus in this case the specified distribution is the distance to the first scattering center.
}

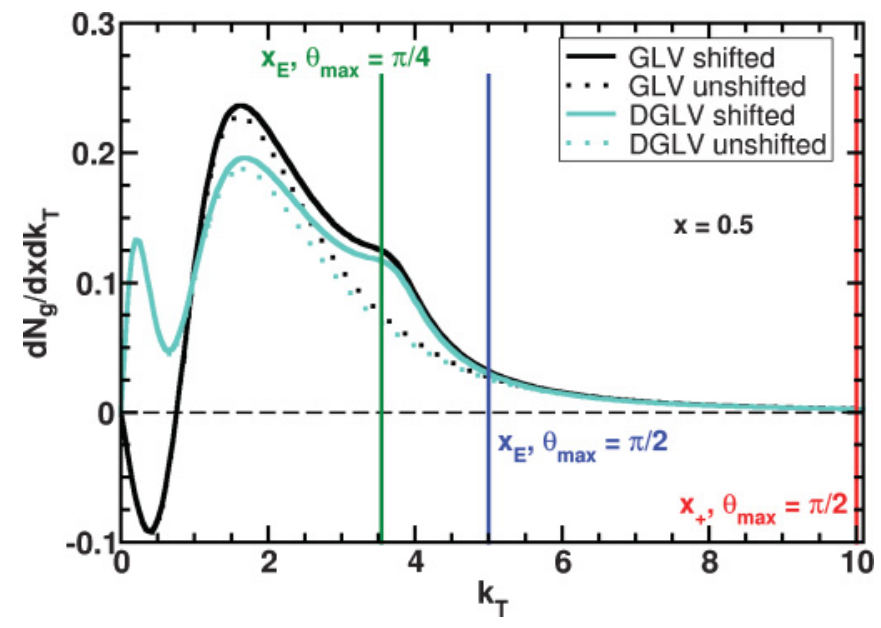

FIG. 3. (Color online) Comparison of $d N_{g} / d x d k_{T}$ spectra as a function of $k_{T}$ with $x=0.5$ between GLV (all masses set to zero) and DGLV (nonzero radiated gluon mass) formulations using both the shifted integrand of Eq. (2) (solid curves) and the unshifted integrand of Eq. (1) (dotted curves). The horizontal dashed line at 0 is meant to guide the eye. The three vertical lines represent the choices of $k_{\max }$ described in the text. $d N_{g} / d x d k_{T}$ better respects the collinear approximation for larger values of $x$, as it has little weight near $k_{T} \sim$ $k_{\max }$. At small $k_{T}$ the radiated gluon mass, surprisingly, enhances radiation for DGLV due to coherence effects; at larger $k_{T}$ the mass has the expected effect of suppressing $d N_{g} / d x d k_{T}$.

it would have support only in the physical regions of the $q_{T}$ and $k_{T}$ integration space. However, due to the small $x$ and collinear approximations, the integrand violates kinematic limits: one can clearly see that the integrand in Eq. (2) has support over all $q_{T}$ and $k_{T}$ (this is also true of the unshifted integrand, Eq. (1)). We enforce physicality in the hope of better approximating the exact result by restricting the $q_{T}$ and $k_{T}$ integration region with cutoffs. A new result from this paper is a quantitative estimate of the systematic uncertainty that results from allowing $\mathcal{O}(1)$ variations of these cutoffs on some observables and extracted medium parameters. Surprisingly, we find that $d N_{g} / d x$ is highly sensitive to variations in the $k_{T} \mathrm{UV}$ cutoff.

For the $q_{T}$ integration we make the usual choices, $q_{\min }=0$ and $q_{\max }=\sqrt{3 \mu E}$. The value for $q_{\max }$ is, in principle, the maximum momentum transfer allowed using relativistic kinematics, though it has been pointed out that this is no longer correct with the change of variables $\mathbf{q} \rightarrow \mathbf{q}+\mathbf{k}$ used in the final steps leading to Eq. (2). ${ }^{4}$ Nevertheless, Eq. (2) is rather insensitive to the $q_{T}$ cutoff [30], and we show later explicit calculations of $d N_{g} / d x d k_{T}$ using the shifted, Eq. (2), and unshifted, Eq. (1), integrands. These comparisons in Figs. 2 and 3 demonstrate that the shifted integrand with the unshifted $q_{\max }$ reproduces well the results of the unshifted integrand, albeit with minor artifacts.

Previous work by BDMS [25] found a strong sensitivity to a phenomenological IR cutoff in the $k_{T}$ integration of the

\footnotetext{
${ }^{4}$ We thank Ulrich Heinz for finding this error in logic.
} 
BDMPS multiple soft scattering calculation, a residual of the original vacuum radiation IR divergence. They also showed that the sensitivity of a quenching factor to variations in this cutoff decreased with increasing parent parton energy. The IR cutoff was imposed to approximate the influence of a nonzero thermal gluon mass; we find that including a gluon mass at the level of the gluon propagators [30] decreases the sensitivity of the energy loss and calculated $R_{A A}$ (see Table I) to the choice of gluon mass. This result is due to a nontrivial cancellation of effects that we will discuss further.

We do find, however, that the radiated gluon spectrum and resulting parton energy loss are extremely sensitive to the choice of the UV cutoff in the $k_{T}$ integration. Furthermore, we find a sensitivity to the particular interpretation of $x$ used in relating the components of $k$ to the components of $p$. It turns out that the derivations of $d N_{g} / d x$ in the literature have used two different, albeit equal to lowest order in collinearity, definitions of $x$. We use the light-cone normalization $p^{ \pm}=$ $p^{0} \pm p^{z}$, with inverse $p^{0, z}=\left(p^{+} \pm p^{-}\right) / 2$, and denote $p^{0}=$ $E$ and $p^{+}=E^{+}$. With these conventions the two definitions of $x$ are (1) the fraction of plus momentum carried away by the gluon $[27,28]$,

$$
x=x_{+} \equiv k^{+} / p^{+}=k^{+} / E^{+} ;
$$

and (2) the fraction of energy carried away by the gluon [29],

$$
x=x_{E} \equiv k^{0} / p^{0}=k^{0} / E .
$$

In the usual notation, where parentheses designate fourmomenta, $k=\left(k^{0}, k^{z}, \mathbf{k}\right)$, square brackets denote light-cone momenta, $k=\left[k^{+}, k^{-}, \mathbf{k}\right]$, and boldfaced variables are the transverse two-vectors $\left(k_{T}=|\mathbf{k}|\right)$, we find that the radiated massless gluon has on-shell momentum

$k=\left(x_{E} E, \sqrt{\left(x_{E} E\right)^{2}-\mathbf{k}^{2}}, \mathbf{k}\right)=\left[x_{+} E^{+}, \frac{\mathbf{k}^{2}}{x_{+} E^{+}}, \mathbf{k}\right]$.

With these definitions of $x_{+}$and $x_{E}$ one may derive the exact relationships,

$$
\begin{aligned}
& x_{+}\left(x_{E}\right)=\frac{1}{2} x_{E}\left(1+\sqrt{1-\left(\frac{k_{T}}{x_{E} E}\right)^{2}}\right), \\
& x_{E}\left(x_{+}\right)=x_{+}\left(1+\left(\frac{k_{T}}{x_{+} E^{+}}\right)^{2}\right),
\end{aligned}
$$

where we have assumed that the initial parent parton momentum is $P=(E, E, \mathbf{0})$, and, hence, $E^{+}=2 E$. These relationships are exact; therefore $x_{+}\left(x_{E}\left(x_{+}\right)\right)=x_{+}$and $x_{E}\left(x_{+}\left(x_{E}\right)\right)=$ $x_{E}$. From the formulas, it is easy to see that in the collinear limit, $x_{+}=x_{E}$, so it is natural that the integrand in Eq. (2) is the same when derived using these two different definitions of $x$ : in one case terms of order $k_{T} / x_{E} E$ are dropped, whereas in the other terms of order $k_{T} / x_{+} E^{+}$are dropped. Thus $d N_{g} / d x_{+} d k_{T} d q_{T}\left(x_{+}\right)=d N_{g} / d x_{E} d k_{T} d q_{T}\left(x_{E}\right)$ in the collinear limit.

There are two justifications for, and there are two derivations of, the $k_{T}$ cutoff in the literature: (1) when interpreting $x$ as $x_{E}, k_{\max }=x_{E} E$ keeps $k$ always real (note that in the $x=x_{+}$ representation, $k$ is real regardless of the value of $k_{T}$ ) [44]; (2) when interpreting $x$ as $x_{+}, k_{\max }=x_{+} E^{+}$enforces forward emission $\left(k^{+} \geqslant k^{-}\right)$[59]. ${ }^{5}$ In this work, we interpret $k_{\max }$ as enforcing consistency with the collinear approximation. It is, then, useful to determine that cutoff from a physical condition independent of the interpretation of $x$; we choose to set $k_{\max }$ by requiring that the emitted radiation be within a cone of angle $\theta_{\max }$ centered on the direction of the parent parton. In this way we may control the collinearity of the radiation by varying the maximum angle of emission allowed. Simple trigonometry relates

$$
k_{\max }= \begin{cases}x_{+} E^{+} \tan \left(\theta_{\max } / 2\right), & x=x_{+}, \\ x_{E} E \sin \left(\theta_{\max }\right), & x=x_{E} .\end{cases}
$$

In particular, when $\theta_{\max }=\pi / 2$

$$
k_{\max }= \begin{cases}x_{+} E^{+}=2 x_{+} E, & x=x_{+}, \\ x_{E} E, & x=x_{E},\end{cases}
$$

and we see that the two original justifications of the $k_{\max }$ cutoff are manifestations of the same physical condition, namely, forward emission. It is worth emphasizing that, with $k_{\max }$ given by Eq. (9), for any numerically equal value of $x_{+}$and $x_{E}$ the integrand in Eq. (2) is integrated out twice as far in $k_{T}$ when interpreting $x$ as $x_{+}$as opposed to when interpreting $x$ as $x_{E}$. We note that a previous study [45] also varied the upper limit of the $k_{T}$ integration. However, that work investigated the radiation into jet cones of various sizes; here we are interested in quantifying the impact of regions of $k_{T}$ space over which there is currently little or no theoretical control.

If the integrand of Eq. (2) respected the assumptions that went into its derivation over the entire integration region, then it would have little weight for $k_{T} \sim x E$. However, Fig. 2 shows that there are values of $x$ for which $d N_{g} / d x d k_{T}$ has large contributions from $k_{T} \sim x E$. In fact, we should expect such a result: the Debye mass $\mu$ is the natural scale for $k_{T}$, and $d N_{g} / d x d k_{T}$ will always have a significant weight at $k_{T} \sim \mu$. As a result, varying $k_{\max } \sim x E$ will always lead to large changes in $d N_{g} / d x$ near $x \sim \mu / E$. In the kinematic regime relevant for RHIC and LHC, numerical study suggests that (1) $d N_{g} / d x(x)$ reaches its maximum at $x \sim \mu / E$ and (2) $d N_{g} / d x d k_{T}\left(x \sim \mu / E, k_{T}\right)$ reaches its maximum at $k_{T} \sim$ $k_{\max }$; for $x \sim \mu / E$ the maximum value of $d N_{g} / d x$ rises quadratically with $k_{\max }$. These observations imply a dramatic sensitivity of $d N_{g} / d x$ - and any quantities derived from it- to the precise choice of $k_{\max }$. Increasing the radiating parton energy decreases the region of $x$ over which $d N_{g} / d x$ is highly sensitive to the cutoff, $x \lesssim \mu / E$. Naively, then, one might expect that increasing $E$ would decrease the sensitivity of observables calculated from $d N_{g} / d x$ to variations of $k_{\max }$. However, we show below that even this expectation does not generally hold.

Figure 3 shows that, as expected, as $x$ increases, the assumption of collinearity, $k_{T} \ll x E$, becomes a better approximation: $d N_{g} / d x d k_{T}$ has little weight near $k_{T} \sim k_{\max }$,

\footnotetext{
${ }^{5} \mathrm{An}$ even more restrictive cutoff for $k_{T}$ results if one also requires forward propagation of the parent parton [7]. Surprisingly this tighter cutoff, which forbids support for $d N / d x$ for $x>1$ and therefore enforces energy conservation, leads to only a small change in $d N_{g} / d x$ [58], and we do not use it here.
} 
and therefore, for these larger values of $x, d N_{g} / d x$ has less sensitivity to the exact choice of $k_{\max }$. One may also observe in Fig. 3 the negative values of $d N_{g} / d x d k_{T}$ for the massless case at small values of $k_{T}$. These negative values are due to the destructive interference between the zeroth-order vacuum production radiation (the QCD analog of the usual $\beta$-decay radiation spectrum from QED) and the first-order medium-induced radiation. The interference is controlled by the ratio of the radiating parton path length $L$ and the coherence length,

$$
\tau_{\mathrm{coh}}=\frac{2 x E}{q_{T}^{2}+\beta^{2}},
$$

found by examining the cosine term in Eq. (2) $\left(q_{T}\right.$ enters $\tau_{\text {coh }}$ here due to the shift in integration variables $q_{T} \rightarrow q_{T}+k_{T}$ upon going from Eq. (1) to Eq. (2)). For larger values of $x$ the coherence length $\tau_{\text {coh }}$ is longer, and the destructive interference more important; hence $d N_{g} / d x d k_{T}$ is negative for small values of $k_{T}$ for the massless results shown in Fig. 3 but not in Fig. 2. Including a nonzero mass for the radiated gluon reduces $\tau_{\text {coh }}$ and, therefore, the influence of the destructive interference. As a result, for small values of $k_{T}$ the $d N_{g} / d x d k_{T}$ spectrum is actually enhanced for the massive compared to the massless case. At larger values of $k_{T}$ the nonzero thermal gluon mass exhibits the expected effect of suppressing bremsstrahlung radiation. Unfortunately, then, introducing a phenomenological $k_{T}$ cutoff in the IR into a massless $d N_{g} / d x$ formula does not capture well the complicated dynamics resulting from allowing the radiated gluons to pick up a nonzero thermal mass from the medium. More readily visible in Fig. 3 than in Fig. 2 is the removal of the minor artifacts from the shift in integration variable when evaluating the unshifted integrand of Eq. (1).

The ambiguity in the interpretation of $x$ has consequences beyond the differences in the corresponding limits of the $k_{T}$ integration. The gluon $d N_{g} / d x$ distributions obtained from Eq. (2) using the two different $x$ definitions imply different gluon energy spectra and, thus, different amounts of jet quenching. These differences will clearly contribute to the systematic uncertainty in the interpretation of experimental data using the opacity expansion formalism and any other energy loss formalism that invokes the same or similar collinear approximations. Furthermore, to better understand the consequences of the different physical pictures used in different formalisms-for example, by comparing the gluon spectra produced by GLV (traditionally interpreting $x$ as $x_{+}$) and BDMPS-Z-ASW ( $x$ as $x_{E}$ )-one wants all results in terms of the same variables. As a first step we examine an "apples-to-apples" comparison between results derived from the two interpretations of $x$ solely within the opacity expansion approach.

There is an additional problem with energy loss calculations that interpret $x$ in Eq. (2) as $x_{+}$: evaluation of the Poisson convolution $P(\epsilon)$, where $\epsilon$ is explicitly an energy fraction. Previous models that assumed the $x_{+}$interpretation in $d N_{g} / d x$ invoked the collinear approximation to directly use $d N_{g} / d x_{+}\left(x_{+}\right)$unmodified as the input for the Poisson convolution. As we have shown, this is a poor assumption.
To compare the two interpretations of $x$ within GLV and to quantify the effect of assuming $d N_{g} / d x_{+} \approx d N_{g} / d x_{E}$ in finding $P(\epsilon)$, we need to transform $d N_{g} / d x_{+}\left(x_{+}\right)$to $d N_{g} / d x_{E}\left(x_{E}\right)$, which requires the use of a Jacobian:

$$
\begin{aligned}
\frac{d N_{g}^{J}}{d x_{E}}\left(x_{E}\right) & \equiv \int^{x_{E} E \sin \left(\theta_{\max }\right)} d k_{T} \frac{d x_{+}}{d x_{E}} \frac{d N_{g}}{d x_{+} d k_{T}}\left(x_{+}\left(x_{E}\right)\right), \\
\frac{d x_{+}}{d x_{E}} & =\frac{1}{2}\left[1+\left(1-\left(\frac{k_{T}}{x_{E} E}\right)^{2}\right)^{-1 / 2}\right] .
\end{aligned}
$$

Note the change in the upper limit of integration in Eq. (11) as dictated by the basic rules of calculus. The Jacobian, Eq. (12), is strictly $>1$ and is singular as $k_{T} \rightarrow x_{E} E$; the competing effects between it and the numerically smaller $k_{\max }$ on $d N_{g}^{J} / d x_{E}$ and energy loss is discussed in further detail below.

\section{QUANTITATIVE COMPARISONS}

We now wish to quantify the effects of the different $x$ interpretations, $k_{\max }$ values, and gluon mass treatments on $d N_{g} / d x$ and its derived quantities, such as $P(\epsilon)$, the medium parameter $d N_{g} / d y$ extracted from $R_{A A}$, and the total radiated gluon multiplicity $\left\langle N_{g}\right\rangle$. We do so by using five implementations of radiative energy loss; see Table II. In the first three calculations we use the $x=x_{+}$interpretation and $\theta_{\max }=\pi / 2$; in the first two we do not include the effect of the $d x_{+} / d x_{E}$ Jacobian. In the first calculation we assume radiated gluons acquire a thermal mass of the order of the Debye scale, $m_{g}=\mu / \sqrt{ } 2$. The second calculation is the same as the first but with $m_{g} \rightarrow 0$. The third is the same as the second but includes the Jacobian transformation from $x_{+}$to $x_{E}$. In the last two calculations we adopt the $x=x_{E}$ interpretation from the start: in the fourth we take $\theta_{\max }=\pi / 2$, and in the fifth, $\theta_{\max }=\pi / 4$.

We show in Fig. 4, top (bottom) panel, the dramatic difference between the "equivalent" $d N_{g} / d x_{E}$ spectra obtained using the two interpretations of $x$ and taking $E=10 \mathrm{GeV}$, $L=5 \mathrm{fm}(L=1 \mathrm{fm}), \mu \approx 0.46 \mathrm{GeV}$, and $\lambda \approx 1.26 \mathrm{fm}$ as representative RHIC values. Also shown in the figure is the large reduction in $d N_{g} / d x$ as $\theta_{\max }$ is reduced from $\pi / 2$ to $\pi / 4$. That reduction in the spectrum demonstrates the importance of large-angle gluon emission despite the assumption of collinearity. Figure 5, top panel, shows the dramatic reduction in $d N_{g} / d x$ with the introduction of a thermal gluon mass for the aforementioned parameter values; the bottom panel shows

TABLE II. Descriptions of the five main radiative energy loss implementations investigated in this work and their names as listed in diagrams. See text for details.

\begin{tabular}{lcccc}
\hline \hline Name & $x$ & Jacobian & $\theta_{\max }$ & $m_{g}$ \\
\hline$x_{+}, m_{g}=\mu / \sqrt{ } 2$ & $x_{+}$ & No & $\pi / 2$ & $\mu / \sqrt{ } 2$ \\
$x_{+}, m_{g}=0$ & $x_{+}$ & No & $\pi / 2$ & 0 \\
$x_{+}\left(x_{E}\right), \theta_{\max }=\pi / 2$ & $x_{+}$ & Yes & $\pi / 2$ & 0 \\
$x_{E}, \theta_{\max }=\pi / 2$ & $x_{E}$ & No & $\pi / 2$ & 0 \\
$x_{E}, \theta_{\max }=\pi / 4$ & $x_{E}$ & No & $\pi / 4$ & 0 \\
\hline \hline
\end{tabular}



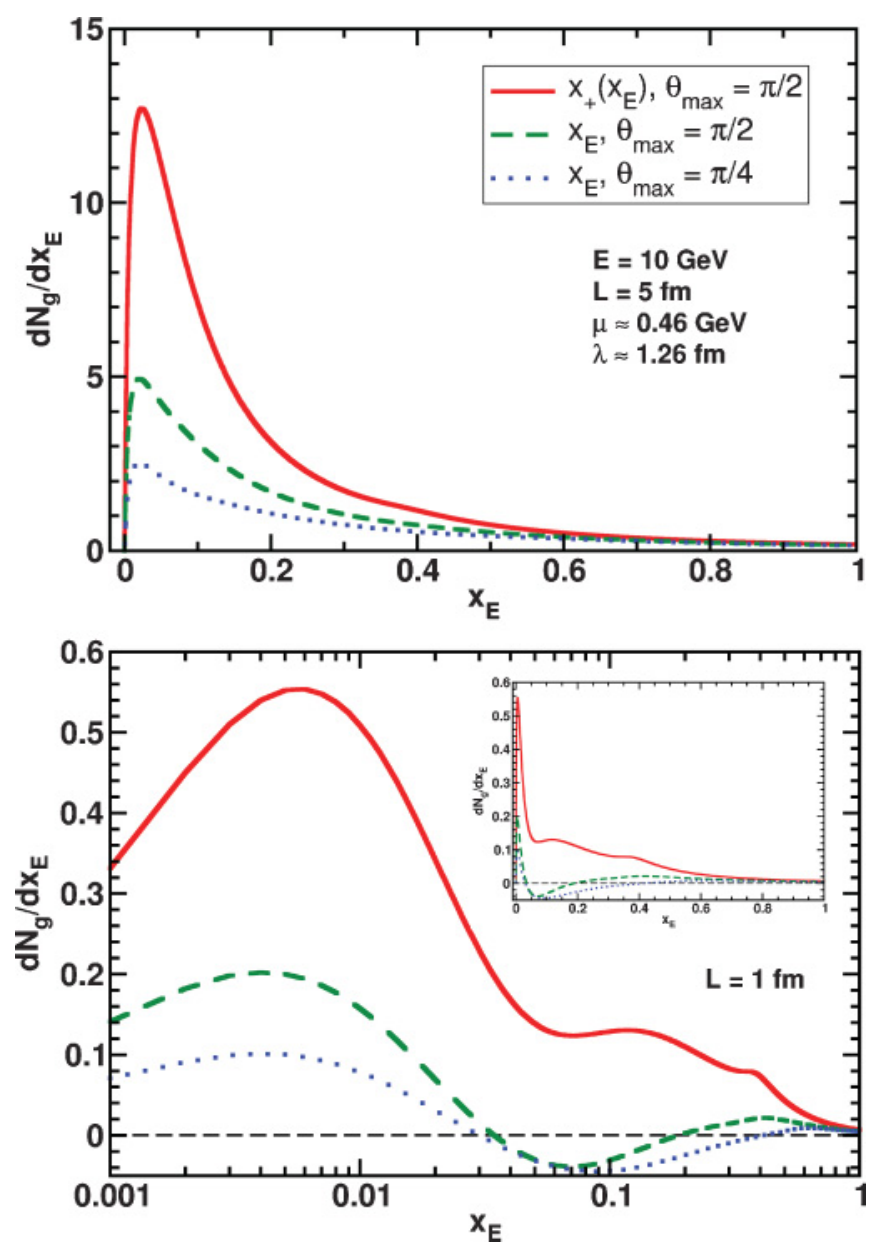

FIG. 4. (Color online) Comparison of $d N_{g} / d x_{E}\left(x_{E}\right)$ for a $10 \mathrm{GeV}$ quark using typical RHIC medium parameters and $L=5 \mathrm{fm}$ (top) or $L=1 \mathrm{fm}$ (bottom) for the collinearly equivalent expressions $d N_{g} / d x$ from Eq. (2) with the $x=x_{E}$ interpretation and $x_{+}\left(x_{E}\right)$ from Eq. (11). Also shown is $d N_{g} / d x$ from Eq. (2) with $x=x_{E}$ and $\theta_{\max }=\pi / 4$, to explore the systematic uncertainty in the choice of $k_{\max }$. The inset (bottom) shows results on a linear scale for clarity.

that for shorter path lengths, such as $L=1 \mathrm{fm}$, the inclusion of a thermal mass for the radiated gluon can actually enhance the emission of bremsstrahlung radiation.

The only difference between the $x_{E}$ and the $x_{+}\left(x_{E}\right)$ curves, theoretically indistinguishable to lowest order in collinearity, in Fig. 4 comes from terms of $\mathcal{O}\left(\left(k_{T} / x_{E} E\right)^{2}\right)$ and higher. Figure 6 quantifies the reduction of the effect of these terms as we decrease $\theta_{\max }$ from $90^{\circ}$ to $30^{\circ}$.

It is also worthwhile to consider the "apples-to-oranges" comparison of the $x_{+}\left(x_{E}\right)$ curve in Fig. 4 and the $x_{+}, m_{g}=0$ curve in Fig. 5 (top panel). In particular, we observe that the former has a reduced peak height at $x \sim \mu / E$ but is larger for higher values of $x$. This generic behavior is due to a nontrivial interplay between the Jacobian, which always multiplies the $d N_{g} / d x_{+} d k_{T}$ integrand by a number $>1$, and the numerically reduced $k_{\max }=x_{E} E$ used in Eq. (11). Figure 3 shows that the collinear approximation is better at larger values of $x$ as demonstrated by the small values of $d N_{g} / d x d k_{T}$ at $k_{T} \sim k_{\max }$ compared to those at lower $k_{T}$.
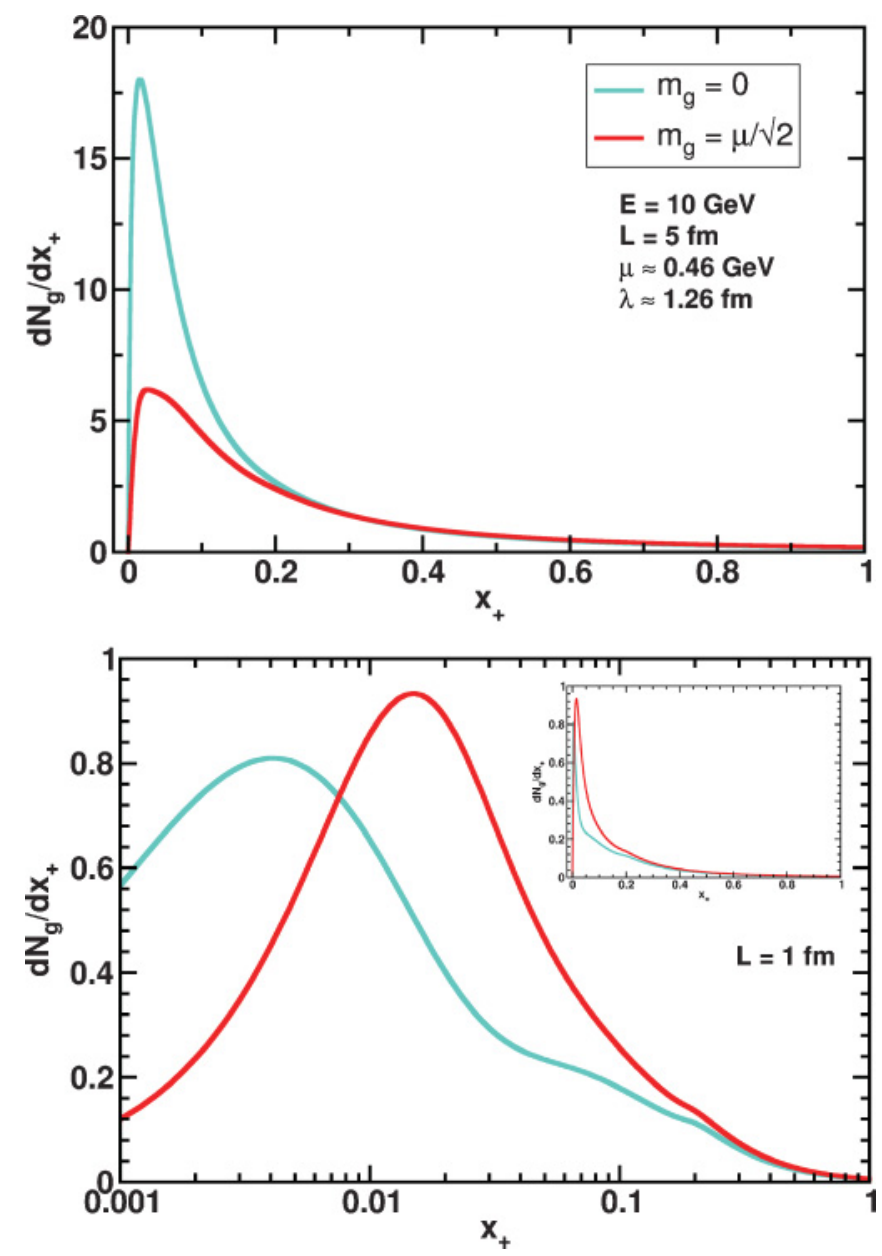

FIG. 5. (Color online) Comparison of $d N_{g} / d x_{E}\left(x_{E}\right)$ from GLV and DGLV $\left(m_{g}=\mu / \sqrt{ } 2\right)$ for an $E=10 \mathrm{GeV}$ quark using typical RHIC medium parameters and $L=5 \mathrm{fm}$ (top) or $L=1 \mathrm{fm}$ (bottom). The inset (bottom) shows results on a linear scale for clarity. Note the enhancement of $d N_{g} / d x$ at $L=1 \mathrm{fm}$ for the massive case.

The Jacobian, though, introduces an integrable divergence at $k_{T}=x_{E} E$, so the $x_{+}\left(x_{E}\right)$ curve is systematically larger than the $x_{+}$curve at larger values of $x$. Since the total gluon yield $\left\langle N_{g}\right\rangle$ must be unmodified by a change in integration variables, the increased yield at larger $x$ must come at the expense of gluon yield at smaller values of $x$; hence, the numerical reduction in $k_{\max }$ wins in this small- $x$ region. This redistribution of probability means that $\langle x\rangle$ from $x_{+}\left(x_{E}\right)$ is always greater than $\langle x\rangle$ from $x_{+}$. For any given set of medium parameters and path length, $\left\langle N_{g}\right\rangle$ must be the same for both the $x_{+}\left(x_{E}\right)$ and the $x_{+}$implementations; because $\langle x\rangle$ is always larger for the former, using $x_{+}\left(x_{E}\right)$ will always produce a smaller $R_{A A}$ than $x_{+}$. For any given $\theta_{\max }, d N_{g} / d x d k_{T}$ with $x=x_{+}$is integrated out to a larger $k_{\max }$ than for $x=x_{E}$. Therefore, the $x_{+}$interpretation will always yield a smaller $R_{A A}$ than the $x_{E}$ interpretation. Using similar reasoning the $x_{E}$ calculation with $\theta_{\max }=\pi / 2$ will always generate a smaller $R_{A A}$ than the $x_{E}$ with $\theta_{\max }=\pi / 4$ calculation. Based on these arguments we expect that the extracted medium parameter $d N_{g} / d y$ will be ordered from smallest to largest according 


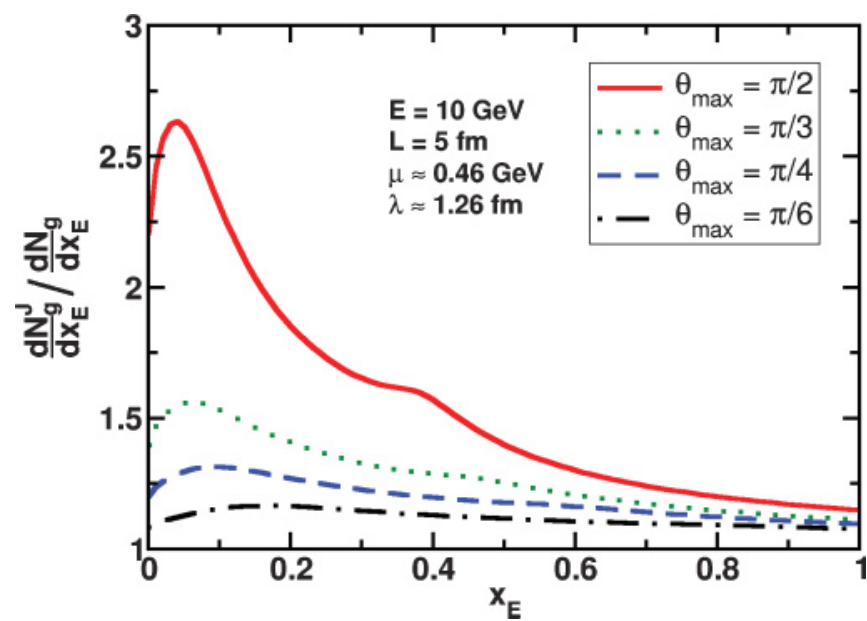

FIG. 6. (Color online) Ratios of gluon spectra obtained from Eq. (11), $d N_{g}^{J} / d x_{E}\left(x_{E}\right)$, to that obtained from Eq. (2) with $x=x_{E}$, $d N_{g} / d x_{E}\left(x_{E}\right)$, as a function of $x_{E}$ for different choices of $\theta_{\max }$. Smaller maximum opening angles produce a decreased sensitivity to the collinear approximation.

to $x_{+}\left(x_{E}\right), x_{+}, x_{E}$ with $\theta_{\max }=\pi / 2$, and $x_{E}$ with $\theta_{\max }=$ $\pi / 4$. The $d N_{g} / d y$ values obtained via the statistical analysis (described later) and listed in Table I demonstrate exactly this ordering.

A crucial ingredient for any jet quenching calculation is $P(\epsilon)$, the probability distribution for the fraction of energy $\epsilon$ radiated by a high- $p_{T}$ parton. Described previously, $P(\epsilon)$ is obtained via a Poisson convolution of the single inclusive radiated gluon spectrum, $d N_{g} / d y$, assuming the independent emission of the multiple radiated gluons. $P(\epsilon)$ can be decomposed into discrete and continuous pieces,

$$
P(\epsilon)=P^{0} \delta(\epsilon)+\tilde{P}(\epsilon)+P^{1} \delta(1-\epsilon) .
$$

$P^{0}$ is the probability of radiating no gluons (and hence no energy loss) and is given by

$$
P^{0}=\exp \left(-\left\langle N_{g}\right\rangle\right) \text {. }
$$

$P^{1}$ encodes the probability "leakage," the probability that the radiating parton loses a fraction of energy greater than unity, that results from the assumption of independent emissions used here. We show in Fig. 7 the $P(\epsilon)$ generated from four of the $d N_{g} / d x$ curves investigated in this work (themselves displayed in Figs. 4 and 5): $x_{+}$with $m_{g}=0, x_{+}\left(x_{E}\right), x_{E}$ with $\theta_{\max }=\pi / 2$, and $x_{E}$ with $\theta_{\max }=\pi / 4$. The ordering of the average fractional energy loss $\langle\epsilon\rangle$ (represented by the vertical lines in Fig. 7) is consistent with the qualitative arguments described in the previous paragraph. As noted previously $\left\langle N_{g}\right\rangle$ is identical for the $x_{+}$and $x_{+}\left(x_{E}\right)$ models; hence, $P^{0}$ is also identical for the two. Previous evaluations of $P(\epsilon)$ using the $x_{+}$ interpretation approximated $d N_{g} / d x_{+}\left(x_{+}\right) \approx d N_{g} / d x_{E}\left(x_{E}\right)$. We find a systematic, though small, difference between the $\langle\epsilon\rangle$ from this approximation and that resulting from the correct transformation of $d N_{g} / d x_{+}$to $d N_{g}^{J} / d x_{E}$; the modest variation in $P(\epsilon)$ also produces only an $\sim 10 \%-20 \%$ change in the extracted $d N_{g} / d y$. The continuous parts of $P(\epsilon), \tilde{P}(\epsilon)$, have the expected ordering: from smallest to largest according to $x_{E}$ with $\theta_{\max }=\pi / 4, x_{E}$ with $\theta_{\max }=\pi / 2, x_{+}$. This ordering
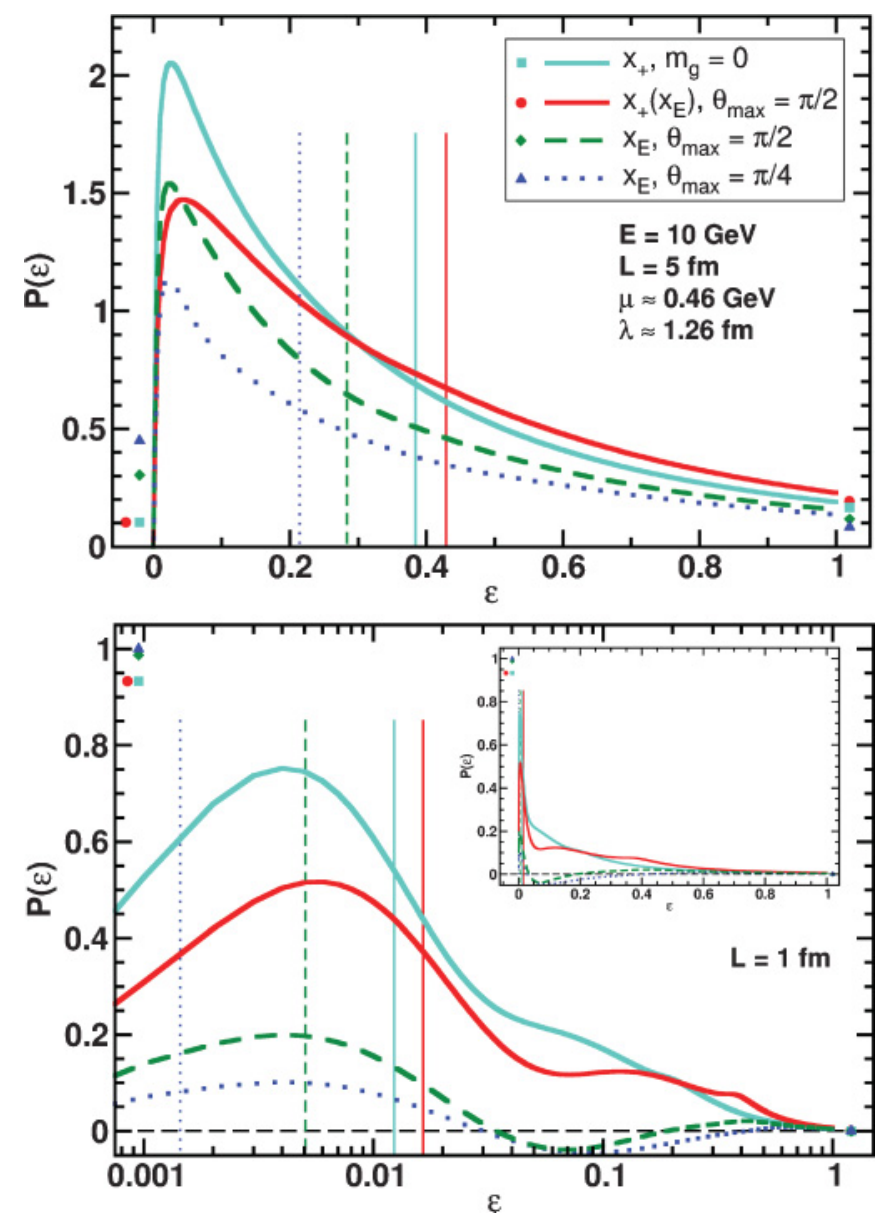

FIG. 7. (Color online) Plots of $P(\epsilon)$ for a $10-\mathrm{GeV}$ quark in typical RHIC conditions with $L=5 \mathrm{fm}$ (top) and $L=1 \mathrm{fm}$ (bottom). $P(\epsilon)$ obtained by convolving the single inclusive spectra $d N_{g} / d x$ shown in Figs. 4 and 5. Vertical lines represent the $\langle\epsilon\rangle$ for each $P(\epsilon)$ distribution. Symbols represent the weight of the $\delta$ functions at $\epsilon=0$ and $\epsilon=1$. The inset (bottom) shows results on a linear scale for clarity.

follows simply from the integration of the $d N_{g} / d x d k_{T}$ curves out to successively larger $k_{\max }$. The nontrivial ordering of $\tilde{P}(\epsilon)$ for the $x_{+}$and $x_{+}\left(x_{E}\right)$ interpretations shows explicitly the redistribution of probability from smaller to larger $\epsilon$ due to the combined effects of the Jacobian, Eq. (12), and the numerically reduced $k_{\max }$ of Eq. (11).

Ultimately, we wish to quantify the effects on extracted medium parameters of (1) the two collinearly equivalent interpretations of $x$, (2) varying of $k_{\max }$ (via $\theta_{\max }$ ), and (3) the thermal mass of the gluon. We focus here on $\pi^{0} R_{A A}\left(p_{T}\right)$ measurements obtained from the 5\% most central $\mathrm{Au}+\mathrm{Au}$ collisions at $\sqrt{ } s=200 \mathrm{AGeV}$ [8]. We follow WHDG [33] in the implementation of energy loss: leading-order pQCD-based production spectra for gluons and light quarks, followed by in-medium energy loss, and, finally, KKP fragmentation into pions. Initial-state effects such as Cronin enhancement and shadowing are neglected; as such the $p_{T}$ dependence of $R_{A A}$ here is stronger than in works that describe the trend of the data better [27]. 
So far in this paper we have only discussed medium-induced gluon radiation, and for one set of $R_{A A}$ calculations we continue to use purely radiative energy loss. However, it is known that for $\mathrm{PQCD}$ in the kinematic regimes appropriate to RHIC and even LHC, elastic scattering results in energy loss of the same order of magnitude as that from radiative energy loss [32,33,60]; collisional energy loss makes a significant contribution to the total leading particle suppression at RHIC and LHC [33,61]. Therefore, we also calculate $R_{A A}$ using convolved inelastic and collisional loss. We use the BraatenThoma calculation $[62,63]$ of the mean elastic energy loss and use the fluctuation-dissipation theorem to estimate the width of the elastic energy loss distribution [33]. These assumptions are a poor approximation for RHIC conditions because the number of collisions is typically too small for the central limit theorem to apply. Hence, Gaussian distributions do not do a good job of representing the actual, highly skewed distributions involved; substantially improved results were derived in Ref. [57]. However, for the purposes of this paper we do not assess the theoretical uncertainties resulting from the treatment of the elastic channel (in particular, we do not vary within reason the kinematics in the collisional calculations); this is a very interesting question in its own right.

For the radiative energy loss calculation, we include the full multigluon fluctuations through the Poisson convolution. We also account for path-length fluctuations due to parent parton production points and trajectories in the medium for both inelastic and collisional loss with an approximate implementation of Bjorken expansion [33]. A standard Glauber modeling of the medium with a diffuse Woods-Saxon nuclear density function is used [33]. Specifically, hard production is assumed to scale with binary collisions; the medium density is assumed to follow participant scaling. As shown in Ref. [26] this geometry yields results in good agreement with those found when using a medium density given by full $3+1$-dimensional ideal hydrodynamics and is sufficient for the purposes of this paper. The strong coupling constant is held fixed at $\alpha_{s}=0.3$. We use thermal field theory to relate $\mu=g T$ and $\lambda=1 / \rho \sigma$, with $\sigma \propto 1 / \mu^{2}$ [33]. The only independent variable left is the single input parameter $d N_{g} / d y$, the rapidity density of gluons in the pure glue QGP assumed here (increasing $d N_{g} / d y$ increases the medium density and decreases $R_{A A}$ ). The rigorous statistical analysis of Refs. [8] and [9] was then used to determine the value and $1-\sigma$ uncertainty of $d N_{g} / d y$ that yields a theoretical curve that "best fits" the data, given the experimental statistical and systematic errors.

We show in Fig. 8 the PHENIX measurement of $\pi^{0}$ $R_{A A}\left(p_{T}\right)$ for the $5 \%$ most central collisions and the best-fit curves to the data for the five different implementations of the radiative energy loss considered in this paper (see Table II) for both purely radiative energy loss (top panel) and convolved radiative and elastic loss (bottom panel). We note that, when compared at similar centrality bins, the STAR [64] measurements of $\pi^{+}+\pi^{-} R_{A A}$ systematically differ from the shown PHENIX [8] $\pi^{0} R_{A A}$ by as much as $\sim 50 \%$. Such a discrepancy represents a potential source of additional systematic uncertainty in extracted medium properties that we do not attempt to evaluate here.
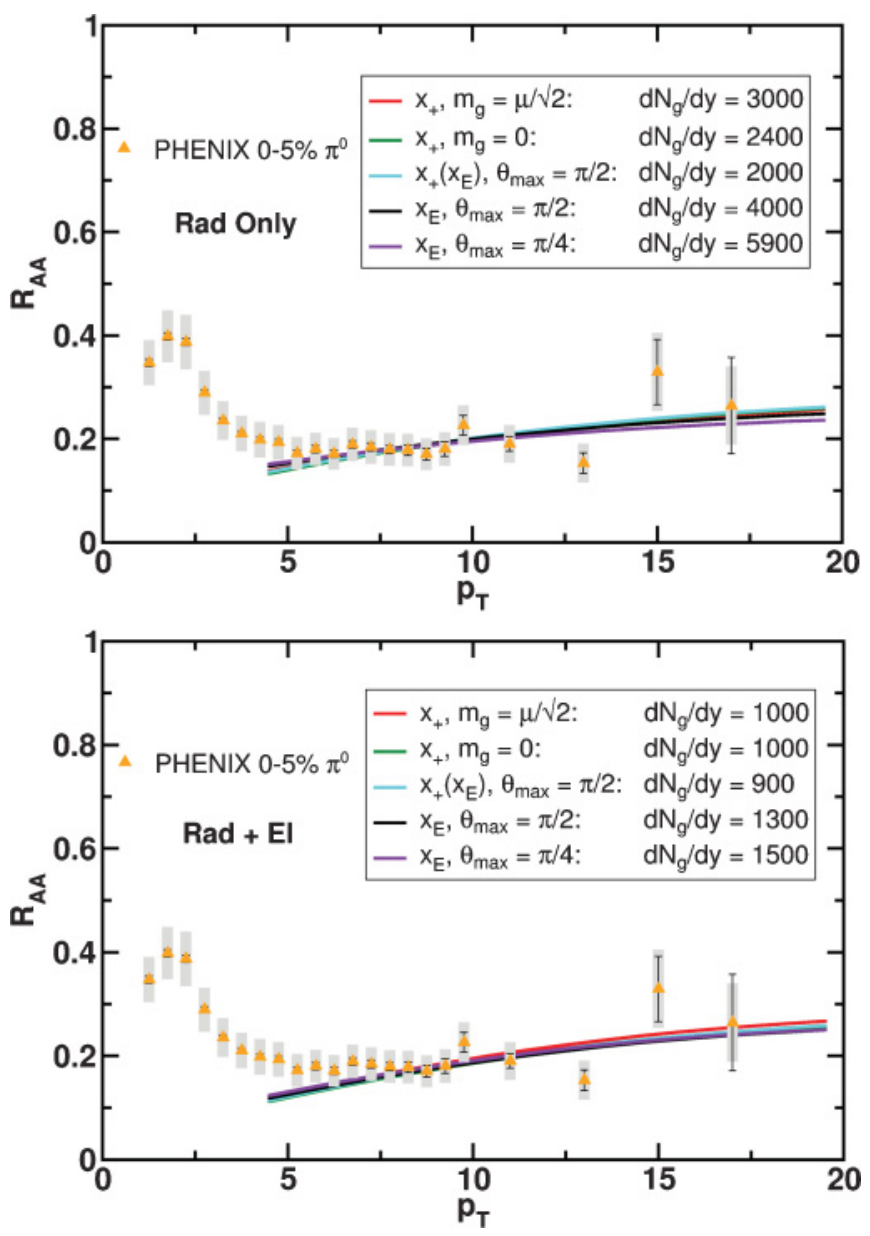

FIG. 8. (Color online) The "best-fit" curves of the five models discussed in the text to PHENIX measurements of the $5 \%$ most central $\pi^{0} R_{A A}$ [8]: top, including only radiative energy loss; bottom, including both radiative and collisional energy loss. Fits performed using the methods of Refs. [8] and [9]. Uncorrelated errors are represented by bars, whereas correlated errors are shown as gray boxes, and there is an overall scale uncertainty of $\pm 12 \%$ not shown.

The results in Fig. 8 indicate that the extracted $d N_{g} / d y$ values for the massive $\left(m_{g}=\mu / \sqrt{ } 2\right)$ and massless $\left(m_{g}=0\right)$ emitted gluon cases are quite similar despite the large differences in $d N_{g} / d x$ for large path lengths (Fig. 5, top panel). As we have shown this behavior is due to the interaction of the effects of a thermal gluon mass on the coherence length and the radiation kernel. As the path length decreases, the difference between the massive and the massless integrated $d N_{g} / d x$ decreases until eventually the massive distribution exceeds the massless one. For central RHIC collisions the results shown in Fig. 5 (bottom panel) indicate that this path length is $\sim 1 \mathrm{fm}$. As noted in Ref. [25] physical observables are quite sensitive to the specific numerical choice of the IR cutoff taken when used to approximate the inclusion of a thermal mass for the radiated gluon. The lack of sensitivity to the particular choice of gluon mass shown in this paper is a pleasant surprise. For the convolved energy loss, the extracted medium density is consistent for massive and massless radiated gluons. For identical values of $d N_{g} / d y$ the massless case 
yields a smaller $R_{A A}$; that the extracted medium parameter is consistent is likely due to the statistical analysis trading off goodness of fit in the normalization of $R_{A A}$ for slightly different $p_{T}$ dependencies.

Addressing the primary goal of this paper, we observe from the results in Fig. 8 that the extracted values of $d N_{g} / d y$ for the radiative-only energy loss models, $x_{+}\left(x_{E}\right)$, $x_{E}$ with $\theta_{\max }=\pi / 2$, and $x_{E}$ with $\theta_{\max }=\pi / 4$, vary by almost $200 \%$. It cannot be overemphasized that the first two cases-for which $d N_{g} / d y$ varies by $\sim 100 \%$-are exactly equivalent under the collinear assumption used in the energy loss derivation. As discussed previously, for any particular $\theta_{\max }, x_{+}\left(x_{E}\right)$ produces the smallest $R_{A A}$. Thus, $x_{+}\left(x_{E}\right)$ with $\theta_{\max }=\pi / 2$ represents a lower bound on $R_{A A}$ for fixed medium parameters. Unfortunately, it is not possible to rigorously define an upper bound to the theoretical uncertainty of $R_{A A}$, as decreasing $\theta_{\max } \rightarrow 0$ makes $R_{A A} \rightarrow 1$. We choose the values of observables calculated from the $x_{E}$ with $\theta_{\max }=\pi / 4$ implementation as a working definition of the upper bound. We consider this $\theta_{\max }$ as representing a reasonable $\mathcal{O}(1)$ variation of the coefficient of the $k_{T}$ cutoff that necessarily increases $R_{A A}$ for a given medium density; the surprise, of course, is just how sensitive $R_{A A}$ is to such a variation of the cutoff. We did not explicitly calculate the combined effects of including a nonzero thermal gluon mass and the Jacobian transformation from the $x_{+}$to $x_{E}$ interpretation. ${ }^{6}$ However, because the gluon mass tends to affect the $d N_{g} / d x d k_{T}$ distribution most at small $k_{T}$, while the Jacobian tends to affect $d N_{g} / d x d k_{T}$ most at large $k_{T}$, we expect the two effects to roughly factorize. It is worth noting that the larger $d N_{g} / d y$ values extracted using the radiative-energy-loss-only models are difficult to reconcile with known RHIC $d N_{c h} / d y$ multiplicities [65].

The last row in Table I lists the values of the medium density extracted when elastic energy loss is considered in addition to inelastic in "best-fit" jet quenching comparisons to data. Note that there is no attempt to quantify the uncertainties in the magnitude of the elastic energy loss; the same collisional energy loss formula is convolved with each different implementation of radiative energy loss. It is clear from Table I that the collisional energy loss is quantitatively a very important contribution to the total energy loss suffered by a high- $p_{T}$ parton: the extracted values of $d N_{g} / d y$ are all reduced by a factor of $\sim 2-4$ when both energy loss channels are exploited. We expect this reduction in the extracted medium parameter to increase as the magnitude of radiative energy loss is decreased compared with the elastic; we see exactly this progression in Table I. As the elastic energy loss is assumed to have infinite precision for the purposes of this paper, the systematic theoretical uncertainty of the extracted value of $d N_{g} / d y$ from the collisional approximation and the uncertainty in the specific value of the thermal gluon mass in the radiative energy loss channel necessarily decrease (to

\footnotetext{
${ }^{6}$ Including both effects simultaneously would cause the transformation functions, Eqs. (6) and (7), to change such that $k_{T}^{2} \rightarrow k_{T}^{2}+m_{g}^{2}$; hence $k_{T}^{2} \rightarrow k_{T}^{2}+m_{g}^{2}$ in the Jacobian, Eq. (12). The singularity in the Jacobian will again occur at the maximum upper limit of integration, now given by $k_{\max }=\sqrt{\left(x_{E E}\right)^{2}-m_{g}^{2}}$.
}
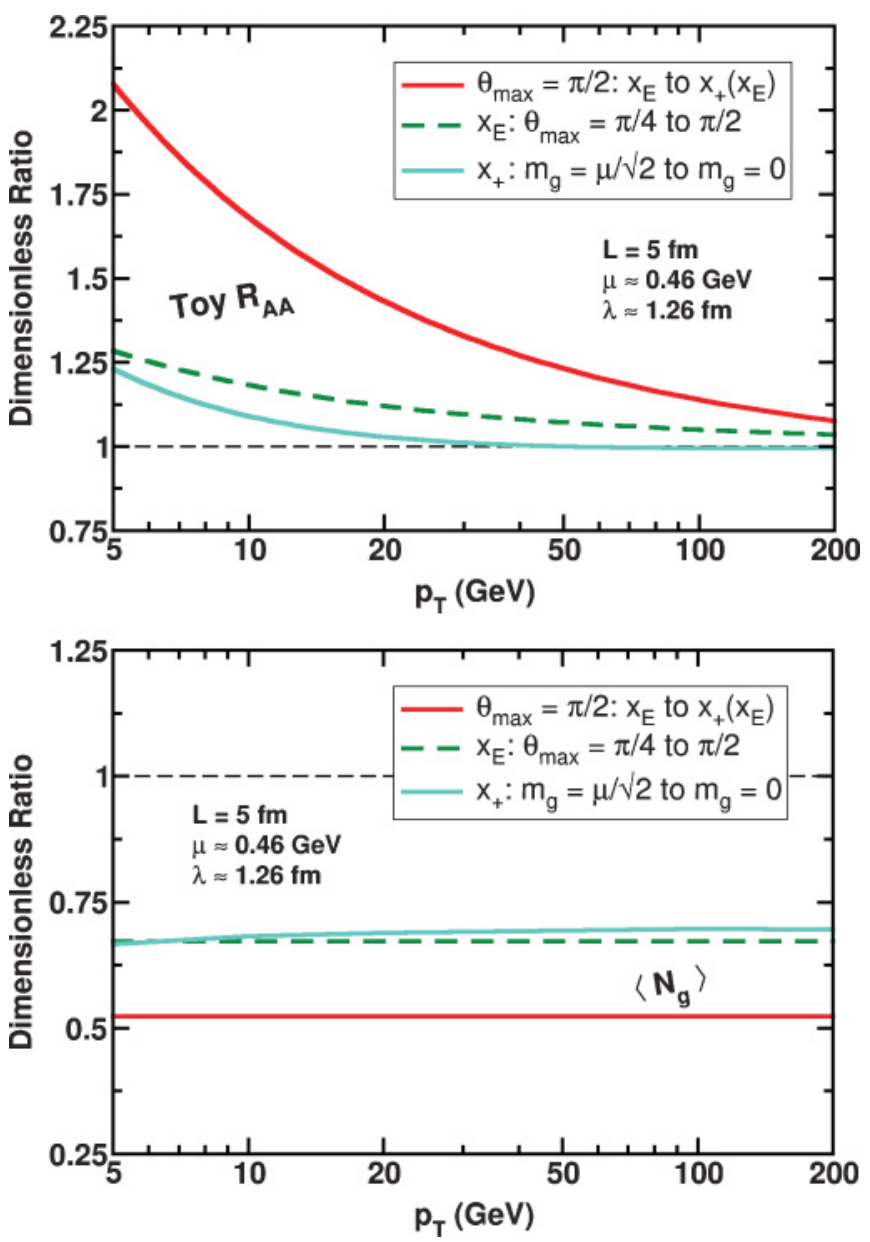

FIG. 9. (Color online) Ratios of a toy $R_{A A}$ model (top) and ratios of the average number of emitted gluons $\langle N\rangle$ (bottom) as a function of parent quark energy for the five models discussed in the text. Collinear and gluon mass effects die out with increasing energy for the toy $R_{A A}$ but do not for $\langle N\rangle$.

about 67\%). One can also see in Table I that both the zero and the nonzero thermal gluon mass cases yield the same extracted value of $d N_{g} / d y$, despite the systematic extra suppression in $R_{A A}\left(p_{T}\right)$ for the zero-mass curve. That the extracted $d N_{g} / d y$ is the same for both is likely due to a trade-off in the statistical analysis in which a better (or worse) fit to the local slope of the data can be compensated for by a worse (or better) fit to the overall normalization.

An important question that must be addressed is whether or not the sensitivity of extracted medium properties to variations in the implementation of the collinear approximation decreases with increasing radiating parton energy. To address this question, we show in Fig. 9 a "toy model" quark partonic $R_{A A}\left(p_{T}\right)$ calculation for an $L=5 \mathrm{fm}$ fixed path length and typical RHIC medium parameters used previously. The sensitivity of the partonic $R_{A A}\left(p_{T}\right)$ to different implementations of the collinear approximation decreases with increasing parton energy. For example, the $\simeq 100 \%$ difference at $5 \mathrm{GeV}$ between the collinearly equivalent $x_{+}\left(x_{E}\right)$ and $x_{E}$ interpretations decreases to $\simeq 10 \%$ at $200 \mathrm{GeV}$. But we observe that the number of emitted gluons $\left\langle N_{g}\right\rangle$ remains 
sensitive to the uncertainties introduced by the collinear approximation; the factor-of- $\sim 2$ difference in $\left\langle N_{g}\right\rangle$ shown in Fig. 9 is nearly energy independent.

The convergence of the ratios of $R_{A A}\left(p_{T}\right)$ with increasing energy can be understood at least partly as a consequence of the small- $x$ pileup in $d N_{g} / d x$. For an energy loss model with $P(\epsilon)$ and a power law production spectrum $d N / d p_{T} \propto p_{T}^{-(n+1)}$, we have that $R_{A A}\left(p_{T}\right) \simeq \int(1-\epsilon)^{n} P\left(\epsilon ; p_{T}\right) d \epsilon$. Then for any two models, say 1 and 2 ,

$$
\frac{R_{A A}^{1}}{R_{A A}^{2}}\left(p_{T}\right) \simeq 1-n\left(\left\langle\epsilon_{1}\right\rangle\left(p_{T}\right)-\left\langle\epsilon_{2}\right\rangle\left(p_{T}\right)\right),
$$

and the ratio of modification factors automatically approaches 1 as the mean energy loss decreases with increasing $p_{T}$. On the other hand, the constancy of $\left\langle N_{g}\right\rangle$ in $p_{T}$ is due to the surprising energy independence of the area under the peak in the $d N_{g} / d x$ distribution. As the energy increases, the distribution becomes more sharply peaked, and its normalization becomes more and more dominated by this energy-independent area.

\section{CONCLUSIONS}

In the Introduction, we laid out the numerous approximations used in deriving the opacity expansion energy loss formulas. In one form or another, these assumptions are at the foundation of the four energy loss calculations that are frequently compared to RHIC data. In particular, these calculations all rely on the lowest-order term in a collinear expansion, where the small parameter is $k_{T} / x E$. Unfortunately, we have found that the opacity expansion energy loss kernel $d N_{g} / d x d k_{T}$ drastically violates the collinear approximation for small values of $x$, the region of $x$ most important for computing many observables, including the leading particle suppression and the average number of emitted bremsstrahlung gluons. While we did not explicitly check this violation for the other energy loss formalisms, it is highly likely they also will be similarly strongly affected by the large-angle radiation that, by assumption of collinearity, is not under theoretical control.

To leading order in collinearity, the two common definitions of $x$ used in energy loss calculations $-x=x_{+}$, the fraction of light-cone plus momentum, and $x=x_{E}$, the fraction of energy taken away by the radiated gluon-are equivalent. We found that for RHIC conditions, the "best-fit" medium density $d N_{g} / d y$ extracted using these two different, but collinearly equivalent, definitions of $x$ varies by a factor of $\sim 2$.

While one may use the $x_{+}\left(x_{E}\right)$ interpretation with $\theta_{\max }=$ $\pi / 2$ as a natural lower bound for the systematic theoretical uncertainty of $R_{A A}$, the upper bound is less obvious. We take the results from the $x_{E}$ and $\theta_{\max }=\pi / 4$ as a working definition; in this case the extracted medium density increases another $\sim 50 \%$ over the $x_{E}$ and $\theta_{\max }=\pi / 2$ model. We note that there is no sense of a "central value" or Gaussian distribution for this uncertainty band: the rigorous notion of a "best" interpretation of $x$ or the "correct" UV cutoff for the lowest-order collinear results does not exist prior to a calculation based on a more exact analytic derivation.

The extracted medium density is a factor of $\sim 2-4$ smaller when collisional energy loss is included in addition to radiative.
The elastic energy loss therefore contributes significantly even for the implementations with the largest radiative energy loss, $x_{+}\left(x_{E}\right)$ with $\theta_{\max }=\pi / 2$. The uncertainties we quote decrease significantly when elastic energy loss is included, although the effects of uncertainty in the collisional channel were not considered here. While the leading particle $R_{A A}$ appears to suffer less systematic uncertainty at LHC energies, the uncertainty in other observables, such as the mean number of emitted gluons, are energy independent. Finally, the effect of a thermal gluon mass on the extracted medium density is surprisingly small due to nontrivial coherence effects. The latter result implies that the specifics of the short-path-length energy loss behavior are very important; future work should, therefore, go beyond the $L \gg \lambda$ approximation.

One of the great debates over the past several years has been the so-called "discrepancy" of extracted medium parameters from the four energy loss models. An oversimplified description would be that the densities found when comparing energy loss models based on the four pQCD formalisms mentioned in the Introduction (GLV, BDMPS-Z-ASW, HT, AMY) differ by a factor of $\sim 4-5[8,9,36]$. It is natural and right to begin any calculation with assumptions about the relevant and irrelevant physics of the problem. However, that the four models include and exclude vastly different physics

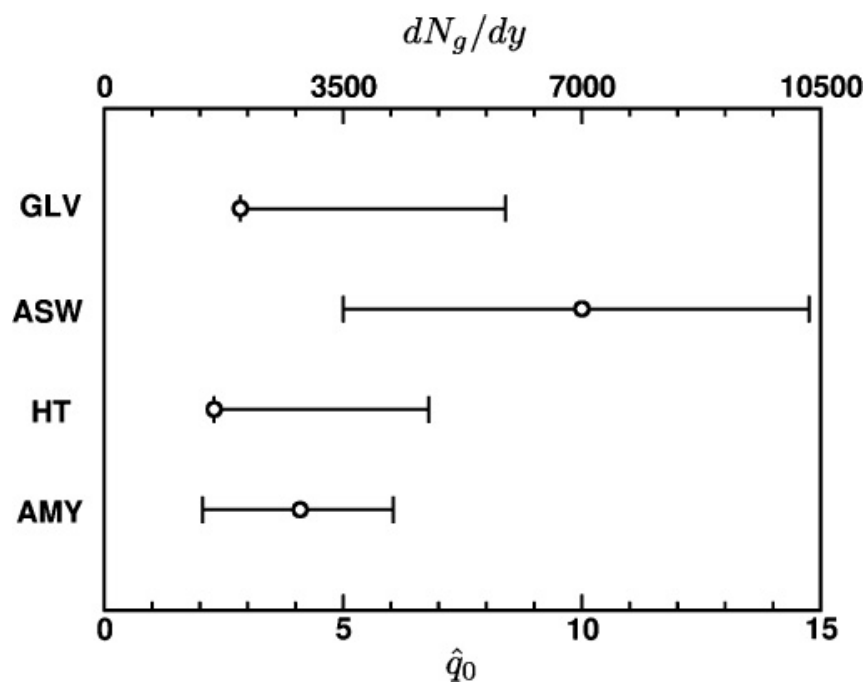

FIG. 10. Schematic comparison of $\hat{q}_{0}$ for the four radiative energy loss formalisms including an estimate of the uncertainty due only to the use of the collinear approximation. The uncertainty bounds are made with the assumption that the other formalisms suffer the same $295 \%$ uncertainty as does the Rad-Only GLV model used here. The open circles for the ASW, HT, and AMY formalisms represent the values of $\hat{q}_{0}$ assuming temperature scaling as reported in Ref. [36]. Since the ASW and AMY formalisms use Minkowski coordinates, the open circles are at the center of the band; because HT uses light-cone coordinates, its open circle is at the left of the band. The extracted $d N_{g} / d y$ values reported in Table I are converted using $\hat{q}=\mu^{2} / \lambda$, similar in spirit to the pure temperature scaling assumed for the other formalisms, with $\tau_{0}=0.6 \mathrm{fm}$; for central RHIC Au $+\mathrm{Au}$ collisions one can approximately convert $1 \hat{q}_{0}$ to $700 d N_{g} / d y$, as shown explicitly on the upper $x$-axis label. The open circle for GLV is set at the left of the band, as previous comparisons with data used light-cone coordinates $[7,33,66]$. 
implies to us that any consistency found must be considered coincidental-even surprising. We see the path forward not in teasing out the origins of the extracted difference but in finding observables that can falsify the basic assumptions about the relevant physics of the quark gluon plasma. Is the medium strongly or weakly coupled? What are its degrees of freedom? If energy loss is perturbative, are parton interactions with the medium better approximated by many soft scatterings or a few hard ones? To answer these questions we must have not only well-controlled experiments but also well-controlled theories. It is, thus, imperative that quantitative estimates be made of the systematic uncertainty introduced into theoretical results from the simplifying assumptions-distinct from the physics assumptions - made in the calculation.

As discussed previously there is no notion of "one standard deviation" of theoretical uncertainty associated with the collinear approximation. Moreover, we have not quantified the consequences of the collinear approximation for the other three energy loss models, although this is both an extremely interesting and a clearly important problem. Nevertheless, we strongly suspect that should this uncertainty be quantified for the other models, then the different values of medium density so far extracted from data would be mutually consistent within the systematic theoretical uncertainties; see Fig. 10. As we have shown in this work, any hope for a quantitative extraction of medium density from high- $p_{T}$ physics at RHIC using the GLV formalism requires a far more careful treatment of noncollinear radiation; this is almost certainly true for the AMY, BDMPSZ-ASW, and HT formalisms too. It is interesting that at LHC the leading particle suppression seems to be a rather collinearly safe observable, whereas the average number of emitted gluons and the spectrum of soft gluons is not. This suggests thatat our current level of theoretical understanding-leading particle observables and jets measured using narrower cones may provide more sensitive tests of jet quenching at the LHC than, for example, full jet reconstruction with large cones.

\section{ACKNOWLEDGMENTS}

The authors wish to thank Miklos Gyulassy, Yuri Kovchegov, and the members of the TECHQM Collaboration for valuable discussions; in particular, we thank Ulrich Heinz and Urs Wiedemann for their help in elucidating the differences between the $x_{+}$and the $x_{E}$ definitions. Additionally, the authors thank Jamie Nagle for quantitatively extracting $d N_{g} / d y$ from PHENIX data and the calculations presented in this work. We also thank Ulrich Heinz for reading and commenting on the manuscript. W.A.H. thanks the Aspen Center for Physics for support during his stay. This work was supported by the Office of Nuclear Physics in the Office of Science of the US Department of Energy under Grant Nos. DE-FG02-05ER41377 and DE-FG02-86ER40281.
[1] M. Gyulassy and L. McLerran, Nucl. Phys. A750, 30 (2005).

[2] W. A. Horowitz and M. Gyulassy, Phys. Lett. B666, 320 (2008).

[3] M. Gyulassy and X. Wang, Nucl. Phys. B420, 583 (1994).

[4] C. P. Herzog, A. Karch, P. Kovtun, C. Kozcaz, and L. G. Yaffe, J. High Energy Phys. 07 (2006) 013.

[5] S. S. Gubser, Phys. Rev. D 74, 126005 (2006).

[6] M. Gyulassy, Lect. Notes Phys. 583, 37 (2002).

[7] M. Gyulassy, P. Levai, and I. Vitev, Phys. Lett. B538, 282 (2002).

[8] A. Adare et al. (PHENIX Collaboration), Phys. Rev. C 77, 064907 (2008).

[9] A. Adare et al. (PHENIX Collaboration), Phys. Rev. Lett. 101, 232301 (2008).

[10] W. A. Horowitz and Y. V. Kovchegov, Phys. Lett. B680, 56 (2009).

[11] J. L. Nagle, Nucl. Phys. A830, 147C (2009).

[12] S. S. Adler et al. (PHENIX Collaboration), Phys. Rev. C 75, 024909 (2007).

[13] G. Lin (STAR Collaboration), J. Phys. G 35, 104046 (2008).

[14] B. I. Abelev et al. (STAR Collaboration), Phys. Rev. C 80, 024905 (2009).

[15] S. Afanasiev et al. (PHENIX Collaboration), Phys. Rev. C 80, 054907 (2009).

[16] A. Adare et al. (PHENIX Collaboration), Phys. Rev. Lett. 98, 172301 (2007).

[17] B. I. Abelev et al. (STAR Collaboration), Phys. Rev. Lett. 98, 192301 (2007).

[18] A. Adare et al. (PHENIX Collaboration), Phys. Rev. Lett. 103, 082002 (2009).

[19] G. Wang (STAR Collaboration), J. Phys. G 35, 104107 (2008).

[20] B. I. Abelev et al. (STAR Collaboration), Phys. Lett. B683, 123 (2010).
[21] W. Horowitz, Nucl. Phys. A783, 543 (2007).

[22] S. Wicks, W. Horowitz, M. Djordjevic, and M. Gyulassy, Nucl. Phys. A783, 493 (2007).

[23] A. Dainese, C. Loizides, and G. Paic, Eur. Phys. J. C 38, 461 (2005).

[24] K. Eskola, H. Honkanen, C. Salgado, and U. Wiedemann, Nucl. Phys. A747, 511 (2005).

[25] R. Baier, Y. L. Dokshitzer, A. H. Mueller, and D. Schiff, J. High Energy Phys. 09 (2001) 033.

[26] N. Armesto, M. Cacciari, T. Hirano, J. L. Nagle, and C. A. Salgado, J. Phys. G 37, 025104 (2010).

[27] M. Gyulassy, P. Levai, and I. Vitev, Nucl. Phys. B571, 197 (2000).

[28] M. Gyulassy, P. Levai, and I. Vitev, Nucl. Phys. B594, 371 (2001).

[29] U. A. Wiedemann, Nucl. Phys. B588, 303 (2000).

[30] M. Djordjevic and M. Gyulassy, Nucl. Phys. A733, 265 (2004).

[31] M. Djordjevic and U. W. Heinz, Phys. Rev. Lett. 101, 022302 (2008).

[32] M. G. Mustafa, Phys. Rev. C 72, 014905 (2005).

[33] S. Wicks, W. Horowitz, M. Djordjevic, and M. Gyulassy, Nucl. Phys. A784, 426 (2007).

[34] H. Zhang, J. F. Owens, E. Wang, and X. N. Wang, Phys. Rev. Lett. 98, 212301 (2007).

[35] G.-Y. Qin, J. Ruppert, C. Gale, S. Jeon, G. D. Moore, and M. G. Mustafa, Phys. Rev. Lett. 100, 072301 (2008).

[36] S. A. Bass, C. Gale, A. Majumder, C. Nonaka, Q. Y. Qin, T. Renk, and J. Ruppert, Phys. Rev. C 79, 024901 (2009).

[37] R. Baier, Y. L. Dokshitzer, A. H. Mueller, S. Peigne, and D. Schiff, Nucl. Phys. B483, 291 (1997). 
[38] R. Baier, Y. L. Dokshitzer, A. H. Mueller, S. Peigne, and D. Schiff, Nucl. Phys. B484, 265 (1997).

[39] B. G. Zakharov, JETP Lett. 63, 952 (1996).

[40] B. G. Zakharov, JETP Lett. 65, 615 (1997).

[41] B. G. Zakharov, Phys. Atom. Nucl. 61, 838 (1998) [Yad. Fiz. 61, 924 (1998)].

[42] U. A. Wiedemann, Nucl. Phys. B582, 409 (2000).

[43] C. A. Salgado and U. A. Wiedemann, Phys. Rev. Lett. 89, 092303 (2002).

[44] N. Armesto, C. A. Salgado, and U. A. Wiedemann, Phys. Rev. D 69, 114003 (2004).

[45] C. A. Salgado and U. A. Wiedemann, Phys. Rev. D 68, 014008 (2003).

[46] N. Armesto, C. A. Salgado, and U. A. Wiedemann, Phys. Rev. Lett. 94, 022002 (2005).

[47] X. Guo and X. N. Wang, Phys. Rev. Lett. 85, 3591 (2000).

[48] X. Wang and X. Guo, Nucl. Phys. A696, 788 (2001).

[49] B.-W. Zhang and X. Wang, Nucl. Phys. A720, 429 (2003).

[50] A. Majumder, E. Wang, and X. N. Wang, Phys. Rev. Lett. 99, 152301 (2007).

[51] P. Arnold, G. D. Moore, and L. G. Yaffe, J. High Energy Phys. 11 (2000) 001.
[52] P. Arnold, G. D. Moore, and L. G. Yaffe, J. High Energy Phys. 11 (2001) 057.

[53] S. Jeon and G. D. Moore, Phys. Rev. C 71, 034901 (2005).

[54] S. Turbide, C. Gale, S. Jeon, and G. D. Moore, Phys. Rev. C 72, 014906 (2005).

[55] A. Kovner and U. Wiedemann, in Quark Gluon Plasma 3, edited by R. Hwa and X. Wang (World Scientific, Singapore, 2003), p. 192.

[56] S. Wicks, arXiv:0804.4704 (2008).

[57] S. Wicks, Ph.D. thesis, Columbia University, 2008.

[58] W. A. Horowitz and B. A. Cole (in preparation).

[59] M. Gyulassy, P. Levai, and I. Vitev, Phys. Rev. Lett. 85, 5535 (2000).

[60] W. A. Horowitz, Ph.D. thesis, Columbia University, 2008.

[61] N. Armesto et al. (eds.), J. Phys. G 35, 054001 (2008).

[62] E. Braaten and M. H. Thoma, Phys. Rev. D 44, 1298 (1991).

[63] E. Braaten and M. H. Thoma, Phys. Rev. D 44, R2625 (1991).

[64] B. I. Abelev et al. (STAR Collaboration), Phys. Lett. B655, 104 (2007).

[65] B. Back et al. (PHOBOS Collaboration), Phys. Rev. C 65, 061901 (2002).

[66] M. Djordjevic, M. Gyulassy, R. Vogt, and S. Wicks, Phys. Lett. B632, 81 (2006). 